\title{
La formación inicial en Didáctica de las Ciencias Sociales según el alumnado egresado
}

\author{
Initial teacher training in Social Sciences \\ Education according to graduate students
}

DOI: $10.7203 / D C E S .41 .21294$

\author{
Sanae Darouech \\ Universidad de Almería, sanaedarouech@hotmail.com \\ María del Mar Felices de la Fuente \\ Universidad de Almería, marfelices@ual.es \\ ORCID iD: https://orcid.org/0000-0003-3236-0177
}

\begin{abstract}
RESUMEN: Este estudio profundiza en la formación inicial para enseñar Ciencias Sociales, con objeto de conocer su impacto en el alumnado universitario, detectar posibles deficiencias e incorporar mejoras. Para ello, hemos establecido dos objetivos: conocer la valoración del alumnado egresado sobre dicha formación e indagar en las competencias que consideran haber adquirido. El enfoque de investigación ha sido mixto, empleando un cuestionario que fue contestado por 62 participantes y una entrevista semiestructurada que se desarrolló con dos maestras recién tituladas. Los resultados muestran una opinión generalmente positiva de la formación recibida, aunque se destacan aprendizajes mayoritariamente teóricos y conceptuales, el uso excesivo de metodologías expositivas, y un nivel de capacitación relativo. Como conclusión, destacamos la necesidad de incorporar nuevos contenidos y enfoques prácticos en la formación inicial en Didáctica de las Ciencias Sociales, a fin de mejorar las competencias profesionales de los futuros docentes.
\end{abstract}

Palabras Clave: formación inicial de profesorado, Didáctica de las Ciencias Sociales, prácticas docentes universitarias, alumnado egresado, metodología de investigación mixta

\begin{abstract}
This research focuses on initial training for teaching Social Sciences, in order to analyse its impact on university students, detect possible deficiencies and incorporate improvements. To this end, we have established two objectives: to examine the graduates'assessment of the received training and to investigate the competences they consider to have acquired. We have used a mixed research methodology, using a questionnaire that was answered by 62 participants and a semi-structured interview that was conducted with two newly graduated teachers. The results show a generally positive opinion of the received training, although highlighting elements such as mostly theoretical and conceptual learning, the excessive use of expository methodologies, and a relative level of acquisition of competences. In conclusion, we underline the need to incorporate new content and practical approaches in the initial teacher training in Social Sciences Education, in order to improve the professional competences of future teachers.
\end{abstract}

KEYWORDS: initial teacher training, Social Sciences Education, university teaching practices, graduate students, mixed research methodology

Fecha de recepción: julio de 2021

Fecha de aceptación: noviembre de 2021

Este trabajo es resultado del Proyecto de Innovación Educativa: “Investigar para Innovar: Desarrollo profesional docente en Didáctica de las Ciencias Sociales" (PIE 19/021), financiado por la Universidad de Málaga; y del Grupo de Innovación Docente "Investigando Prácticas Docentes Universitarias Innovadoras en Didáctica de las Ciencias Sociales" (21_22_1_06C), financiado por la Universidad de Almería. 


\section{INTRODUCCIÓN}

En la actualidad, la enseñanza de las Ciencias Sociales debe ir encaminada a construir ciudadanía democrática y crítica (Gómez, Souto y Miralles, 2021), capaz de interpretar su mundo e intervenir en él para transformarlo. Otros autores como Pagés (2019), amplían este marco señalando que debe favorecer la educación política, así como una visión completa y crítica del mundo, donde se tengan en cuenta la totalidad de espacios geográficos, de hechos históricos y sociales, y la perspectiva local y global de las problemáticas.

Simplificando, estas son algunas de las finalidades actuales que se atribuyen a la enseñanza del conocimiento social. No obstante, atender a estos propósitos debe hacerse a partir de enfoques metodológicos que incidan en la problematización del contenido social, la conexión con temas controvertidos, pasados y presentes (Santisteban, 2019), y el protagonismo activo del alumnado en su propio proceso de aprendizaje.

Dada la importancia que tiene la enseñanza de las Ciencias Sociales para situarse en un mundo colmado de incertidumbre (Pérez, 2019), es necesario interrogarse acerca de qué tipo de formación inicial requieren los docentes que van a guiar al alumnado en el proceso de construcción de su pensamiento social. Asimismo, cabe cuestionarse también si los profesionales de la educación están capacitados para enseñar Ciencias Sociales desde una óptica crítica y emancipadora, sobre todo, por lo que respecta a los estudiantes recién egresados de los grados universitarios. Conocer su capacitación y el impacto de la formación inicial que han recibido resulta clave para saber si esta ha sido significativa y si procurará cambios en la enseñanza del contenido social que favorezcan la formación de una ciudadanía democrática (Arana, Gutiérrez y Pagès, 2018).

Sabemos que la formación inicial que reciben los futuros y futuras maestras durante su etapa universitaria es transcendental para generar cambios epistemológicos que transformen sus concepciones acerca de las Ciencias Sociales y sus finalidades (González y Fuentes, 2011). Autores como Sanmartí (2007) o Estepa (2017), justifican la necesidad de una formación de calidad basándose en la idea de que un docente, cuando se enfrenta por primera vez a la enseñanza, suele basar su metodología en modelos ya experimentados, o bien, en sus recuerdos como alumno/a, reproduciendo así prácticas de enseñanza vivenciadas anteriormente. De este modo, ofrecer una adecuada formación inicial implica un punto de inflexión para la mejora de la praxis docente en las aulas y la ruptura con rutinas escolares tradicionales (Parra y Fuertes, 2019).

Hace casi dos décadas, Raimundo Cuesta (2003) reivindicaba la importancia de formar al futuro profesorado en una didáctica crítica, que implicara entre otros aspectos, problematizar el presente, pensar históricamente, aprender dialogando e impugnar los códigos pedagógicos y profesionales para cuestionar las bases disciplinares del conocimiento escolar que dificultan las innovaciones y el cambio. En esta línea, autores como Iranzo (2018) han sintetizado las necesidades formativas en elementos como dotar a los profesionales de actitud crítica y activa en su profesión, que los lleve a mejorar tanto sus prácticas educativas como la sociedad que les rodea procurándoles competencias y conocimientos relevantes.

Pese a estas propuestas y la importancia de la formación inicial del profesorado como línea de investigación en el marco de la Didáctica de las Ciencias Sociales -siendo uno de los ámbitos más trabajados (Gómez-Carrasco, López-Facal y Rodríguez-Medina, 2019: 79)-, lo cierto es que sabemos poco acerca de las prácticas formativas universitarias en esta área y el impacto que provoca en los futuros docentes (Pagès y Santisteban, 2014), a excepción de algunos trabajos que se han aproximado a ello (Moril, 2011; Aranda y López, 2017; Chaparro, 2021). En consecuencia, consideramos relevante profundizar en este aspecto y autoinvestigar nuestras propias prácticas, ya que, como docentes universitarios, tenemos el gran desafío de formar a los maestros y maestras que educarán a la ciudadanía del mañana, en una sociedad cada vez más compleja y desafiante (Montero, Martínez y Colén, 2017). 
Tomando en cuenta los estudios con que contamos sobre prácticas formativas universitarias, podemos deducir que la formación de docentes apenas favorece estrategias metodológicas que impliquen cuestionarse o hacerse preguntas (Santisteban, 2015), siendo esta poco reflexiva (Estellés, 2015), lo que se contradice con la propia naturaleza reflexiva del contenido social. Por su parte, Esteban (2019) subraya la escasa preocupación del profesorado por motivar y atraer a su alumnado hacia las materias que imparte, mientras que autores como Moreno (2015) o Vaillant y Marcelo (2015) refuerzan estas ideas, definiendo la didáctica universitaria como una enseñanza tradicional, excesivamente magistral y centrada en la teoría, que difícilmente prepara a los futuros docentes para su práctica profesional. Esta situación es algo más preocupante si atendemos al estudio de Rey (2007), quien señalaba la insuficiente adquisición de competencias, por parte del estudiantado, una vez finalizados sus estudios. En sus investigaciones concluye que la mayoría de los egresados afirma no sentirse suficientemente preparado para enfrentarse a la realidad del aula, manifestando una sensación de insatisfacción al concluir su formación.

Otro ámbito en el que los maestros y maestras recién licenciados encuentran problemas reside en el momento de seleccionar qué enseñar y cómo enseñarlo. En el primer año de ejercicio los docentes acceden a la enseñanza con las teorías, métodos y creencias adquiridas durante el proceso de formación, pero cuando se encuentran frente a un aula, son conscientes de lo complicado que es llevar a la práctica lo aprendido, entendiendo así que, en muchas ocasiones, la formación universitaria tiende a idealizar la enseñanza. Como consecuencia de ese enfrentamiento con la realidad, se produce, lo que González (2012) denomina "shock de la realidad", momento que se caracteriza por un miedo y un estrés sobresaliente. Se produce entonces un proceso de enseñanza basado en el ensayo-error, en el que el/la docente comienza a probar diferentes métodos para intentar responder a las exigencias del aula. Asimismo, en ese primer contacto, se da una confrontación entre lo que el docente cree que debe ser la enseñanza según la formación recibida y la conducta que se adquiere para enfrentar esa enseñanza. Ante esa confrontación surge lo que Marcelo (1991) señaló como la "curva del descenso", en la que se dejan atrás las teorías aprendidas sobre el modo de enseñar y comienza a decaer la ilusión por ser innovador. En este momento, se tiende a utilizar metodologías más tradicionales que habitualmente se van asentado a medida que van transcurriendo los años de ejercicio, obstaculizando así la implementación de nuevas prácticas.

Teniendo en cuenta lo hasta aquí expuesto, la importancia de la formación inicial para transformar la praxis docente y los escasos estudios realizados en el marco de la Didáctica de las Ciencias Sociales sobre la docencia universitaria, con esta investigación profundizamos en la valoración que hace el alumnado egresado -futuro profesorado- sobre su formación y el impacto que ha tenido para favorecer el desarrollo de sus competencias profesionales. Nuestra intención es contribuir a la mejora de las prácticas formativas, puesto que los resultados nos permitirán identificar fortalezas y debilidades, e integrar mejoras en la acción docente desarrollada. Indagamos, por tanto, en una formación que, en el contexto de la Universidad de Almería, para el caso del Grado en Educación Primaria, se estructura en dos asignaturas impartidas en segundo y tercer curso, siendo la primera de ellas de carácter cuatrimestral y obligatoria, con 6 créditos ECTS (Didáctica de las Ciencias Sociales I); y la segunda, anual y también obligatoria, de 9 créditos ECTS (Didáctica de las Ciencias Sociales II). La formación ofrecida en estos cursos se complementa, pues mientras la primera asignatura se centra principalmente en cuestiones epistemológicas, conceptuales y curriculares, así como en el análisis de las finalidades de las Ciencias Sociales, sus contenidos y la formación del pensamiento social; la segunda profundiza de forma más extensa en la enseñanza y el aprendizaje del tiempo y el espacio, la formación de la Ciudadanía Democrática o el patrimonio como elemento trascendental para abordar las Ciencias Sociales. Por lo que respecta al Grado en Educación Infantil, la formación en Didáctica de las Ciencias Sociales se concentra en un único curso, tercero, donde se imparte una asignatura obligatoria anual, denominada Aprendizaje de las Ciencias Sociales en Educación Infantil, que dispone de 9 créditos ECTS. En todos los casos, las asignaturas de ambos 
Grados combinan metodologías expositivas y activas, junto a diversas actividades de aprendizaje y evaluación.

\section{Metodología}

\subsection{Diseño de investigación}

La metodología que se ha empleado posee un carácter mixto, dado que combina enfoques y técnicas cuantitativas y cualitativas. En este caso, el diseño se enmarcar en una tipología explicativa (Creswell y Plano, 2007; Martínez, Castellano y Chacón, 2014), pues primero se recogieron y analizaron los datos cuantitativos y luego se desarrolló la fase de estudio cualitativo que ha ayudado a complementar los resultados cuantitativos iniciales.

Se ha utilizado un diseño cuantitativo no experimental tipo encuesta, aplicando un cuestionario con escala Likert (1-5). Se optó por la investigación mediante encuesta por su versatilidad, eficiencia y generalización (Hernández y Maquilón, 2010). Por otro lado, se integró un diseño cualitativo a través de entrevistas semiestructuradas en profundidad. La combinación de ambas técnicas e instrumentos nos ha permitido en una primera fase, a través del cuestionario, recabar un mayor número de opiniones $\mathrm{y}$, en la segunda, profundizar en determinados aspectos de la investigación a través de las entrevistas.

\subsection{Objetivos}

El objetivo general del estudio es investigar la calidad y el impacto de la formación inicial para enseñar Ciencias Sociales en las aulas. A su vez, este objetivo se ha dividido en dos objetivos específicos:

O.E.1. Conocer la valoración que hace el alumnado egresado sobre la formación inicial recibida en el ámbito de la Didáctica de las Ciencias Sociales.

O.E.2. Indagar en las competencias que consideran haber adquirido para enfrentarse a la enseñanza de las Ciencias Sociales.

\subsection{Participantes}

En la primera fase cuantitativa de la investigación han participado 62 maestros y maestras $(\mathrm{n}=62)$, de los cuales 52 están recién licenciados y 10 son maestros y maestras nóveles, que se encuentran en su primer año como docentes. En todos los casos, son estudiantes egresados de la Universidad de Almería, que han finalizado sus estudios en los últimos tres cursos académicos, en los Grados en Educación Infantil y Educación Primaria. La muestra ha sido seleccionada siguiendo técnicas de muestreo no probabilístico por conveniencia, ya que se atendió al criterio de accesibilidad a los sujetos y de adecuación a los fines de la investigación (McMillan y Schumacher, 2005).

En cuanto al sexo, 50 participantes son mujeres $(80,6 \%)$ y 12 hombres $(19,4 \%)$. Por lo que respecta a la edad, se trata de una muestra joven, pues la media se encuentra entre 22-25 años, siendo más significativo el grupo de edad de 23 años, que representa el $24,2 \%$ del total.

En la titulación de los participantes se observa, como ocurre en el sexo, la prevalencia de un grupo respecto a otro, pues el 77,4\% (48 personas) pertenecen al Grado en Educación Primaria, mientras que el 22,6\% (14 personas) proceden del Grado en Educación Infantil. Se ha optado por contar con respuestas de estudiantes de ambas titulaciones para obtener una visión más amplia de las opiniones sobre la formación inicial que se ofrece para la enseñanza de las Ciencias Sociales.

Adentrándonos en la fase cualitativa del estudio, la selección de las personas participantes en la entrevista semiestructurada se ha realizado a partir de quienes contestaron la encuesta, tomando en cuenta criterios como la accesibilidad y la diversidad. Se intentó que las personas entrevistadas 
perteneciesen a los dos grupos que conforman la muestra: quienes no han ejercido aún la docencia y quienes están en su primer año de ejercicio. Finalmente, de quienes se ofrecieron de forma voluntaria a participar en las entrevistas, se seleccionaron dos personas que cumplían con los criterios referidos, siendo en ambos casos graduadas en Educación Primaria. Una de ellas finalizó sus estudios hace 3 años y está en su primer curso como docente (entrevistada 1, en adelante E1). La segunda persona finalizó el Grado en el curso académico 2019-2020, no habiendo ejercido todavía como maestra (entrevistada 2, en adelante E2).

\subsection{Instrumentos}

Los datos cuantitativos se han extraído a partir de un cuestionario diseñado ad hoc, con preguntas de escala de valoración cerrada tipo Likert (1-5), titulado "Cuestionario sobre valoración de la formación inicial recibida para la enseñanza de las Ciencias Sociales y adquisición de competencias docentes", que ha sido validado por dos jueces expertos y fue difundido a través de la herramienta Formularios de Google, con objeto de tener mayor alcance. Consta de tres bloques y un total de 10 preguntas que recogen a su vez 44 ítems que debían valorarse desde 1 (muy en desacuerdo) hasta 5 (muy de acuerdo). El primer bloque contiene datos de identificación, relativos a la edad, sexo, universidad de procedencia (aunque en todos los casos ha sido la Universidad de Almería) y situación actual; un segundo bloque, recoge información sobre la valoración de la formación recibida en el marco de las asignaturas de Didáctica de las Ciencias Sociales durante la etapa universitaria; por último, en el tercer bloque se solicita a los/as participantes que valoren las capacidades y competencias que consideran haber adquirido para enseñar Ciencias Sociales.

Los datos de carácter cualitativo se han recogido mediante una entrevista semiestructurada, compuesta por 15 preguntas, divididas en dos partes. Una primera, relativa a la valoración de la enseñanza, contiene 6 preguntas encaminadas a conocer el impacto que ha tenido la formación y la valoración que se hace de la labor del profesorado universitario. La segunda parte está compuesta por 9 preguntas que exploran el nivel de competencias que las entrevistadas consideran haber adquirido para enseñar esta materia, haciendo especial hincapié en las dificultades u obstáculos que contemplan para ello.

La entrevista fue igualmente revisada por un juez experto que sugirió diversos cambios y modificaciones para ajustar el instrumento al objeto de estudio. Seleccionadas las participantes, comenzó el proceso de negociación e información sobre el tipo de investigación que se iba a llevar a cabo. Las entrevistas tuvieron lugar en días distintos y duraron una hora y una hora y media, respectivamente. Ante la situación de alarma sanitaria provocada por el COVID-19, se realizaron a través de la plataforma Google Meet, que permitió grabarlas para su posterior transcripción y análisis.

\subsection{Procedimiento de análisis de datos}

Para analizar los datos cuantitativos se ha llevado a cabo un análisis estadístico descriptivo y de frecuencias, con el programa estadísticos SPSS. Asimismo, se ha calculado el Alfa de Cronbach que permite estimar la fiabilidad de un instrumento. En nuestro estudio, el Alfa de Cronbach ha sido de ,908, por lo que podemos hablar de un índice de fiabilidad excelente (George y Mallery, 2003).

Para el análisis de la información cualitativa recogida a través de las entrevistas, hemos empleado como técnica el análisis de contenido (Piñuel, 2002; Blasco y Mengual, 2010), con el fin de reducir y clasificar la información. Siguiendo a Bonilla y López-Suárez (2016), se ha comenzado con una codificación abierta, tratando de localizar conceptos esenciales a los que se les asignó un código. Seguidamente, se continuó con el proceso de categorización, donde los códigos surgidos en la codificación abierta fueron organizados en familias semánticas, dando lugar a la creación de categorías, que a su vez se encuentran conformadas por subcategorías. El proceso de categorización fue de tipo inductivo, pues a partir de la información de las entrevistas se crearon las categorías. 
Por último, se llevó a cabo una codificación axial, en la que se compararon de forma reiterada los datos obtenidos en las entrevistas. Dicha comparación permitió observar características similares entre los códigos y las categorías establecidas previamente en la codificación abierta. La observación de dichas similitudes, donde se producían repeticiones de significados, permitió agrupar los códigos y las categorías, surgiendo de este modo una nueva categorización.

Para garantizar la fiabilidad y validez de los datos se llevó a cabo una triangulación metodológica, siguiendo a Aguilar y Barroso (2015). Dentro de este tipo de triangulación, en nuestra investigación se ha empleado la triangulación entre métodos, donde se han utilizado métodos tanto cuantitativos como cualitativos, para apreciar si de ambos se extraen los mismos resultados, siendo estos coincidentes.

\subsection{Consideraciones éticas sobre el estudio}

Teniendo en cuenta el objeto de estudio y la sensibilidad de algunos de sus contenidos, puesto que recoge opiniones del alumnado egresado y de docentes en activo, las cuestiones éticas se han contemplado cuidadosamente en esta investigación. Para ello, antes de proceder a difundir el cuestionario entre los y las participantes, se les informó a través de correo electrónico sobre los objetivos de este trabajo, el procedimiento de recogida de información y la posibilidad de consultar el informe final de investigación, en caso de publicarse trabajos científicos derivados de los resultados alcanzados. Asimismo, antes de proceder a realizar el cuestionario, en un apartado inicial recogido dentro del mismo, se les informó igualmente de todas estas circunstancias, así como del anonimato y confidencialidad de las respuestas y la voluntariedad de participar en el estudio, pudiendo optar por no participar o por dejar de completar el cuestionario en cualquier momento. Para el caso de las entrevistas, se informó de nuevo a las participantes sobre el proceso de la investigación, el carácter voluntario de las mismas, y el derecho a dejar de participar en ellas en cualquier momento. En ambos casos, se contó con el consentimiento informado de las dos entrevistadas. Cabe señalar que no existió ningún conflicto de intereses entre los participantes y las investigadoras, puesto que la muestra ha estado formada por estudiantes egresados de diferentes cursos académicos, con los que no se guardaba relación.

\section{RESULTADOS}

\subsection{Resultados del análisis cuantitativo}

Adentrándonos en el análisis cuantitativo, y atendiendo a los objetivos que nos planteábamos en este estudio, por lo que respecta al primero de ellos, sobre la valoración que hacen los participantes acerca de la formación inicial recibida para la enseñanza de las Ciencias Sociales, observamos que un $59,7 \%$ manifiesta que la enseñanza recibida les parece adecuada o muy adecuada; el $24,2 \%$ la valora como media; y un 16,1\% la considera poco adecuada o muy poco adecuada. Si analizamos los resultados en relación con el Grado al que pertenecen los participantes, se constata que los procedentes del Grado en Educación Infantil poseen opiniones más diversas, mientras que los estudiantes del Grado en Educación Primaria mantienen cierta tendencia a calificar la enseñanza como adecuada.

Para profundizar más en la valoración de la enseñanza recibida, se incluyó otra pregunta destinada a calificar el nivel de impacto de la formación para su futuro desempeño docente. Las opiniones sobre este ítem revelan que un $49,9 \%$ considera que la enseñanza ha tenido un impacto adecuado o muy adecuado; mientras que el $50,1 \%$ ha valorado el impacto de la formación como intermedio $(24,2 \%)$, reducido $(24,2 \%)$ o muy reducido $(1,7 \%)$, cifras que denotan cierto escepticismo hacia el poder formativo de las prácticas docentes universitarias. Por otro lado, comparando el impacto de la enseñanza por Grados, se aprecia que los resultados no son iguales en ambos, pues, 
aunque las calificaciones que se asignan son diversas, encontrando valores del 1 al 5, el Grado en Educación Primaria posee mayor representación del valor "adecuado" (valor de 4), manifestando así más satisfacción con la formación recibida.

En cuanto a la utilidad de la enseñanza para la futura práctica docente, un 33,8\% considera que los contenidos recibidos durante la carrera les serán útiles o muy útiles; un $38,7 \%$ se muestra dudoso ante la utilidad de lo aprendido y un $27,5 \%$ considera que le servirá de poco o muy poco. Se evidencia así que más de la mitad del alumnado participante en esta investigación $(66,2 \%)$ cuestiona la utilidad de la formación inicial recibida, tendencia que se repite en ambos Grados, lo que nos hace replantearnos las prácticas docentes universitarias, sus enfoques y en qué medida habilitan a los futuros y futuras docentes para enseñar Ciencias Sociales desde los planteamientos requeridos en la actualidad.

En relación con el nivel de satisfacción con la labor docente del profesorado de las asignaturas de Didáctica de las Ciencias Sociales, los resultados revelan que un 59,7\% está satisfecho o muy satisfecho con la labor de sus docentes; un $27,4 \%$ se muestra neutral, indicando que no está ni contento ni descontento, mientras que el $12,9 \%$ afirma estar poco o muy poco satisfecho. Vemos, pues, que casi el $60 \%$ de la muestra valora positivamente la acción docente del profesorado universitario de Didáctica de las Ciencias Sociales.

Se incluyó asimismo un ítem para valorar el tipo de conocimiento que se ha fomentado durante el proceso de formación. Al respecto, el 75,8\% de los participantes considera la enseñanza como teórica o muy teórica, el $16,1 \%$ se mantienen en posturas intermedias, y solo el $8,1 \%$ cree que es poco o muy poco teórica. Estos resultados, similares también en ambos Grados, denotan claramente que la enseñanza que se imparte desde el área de Didáctica de las Ciencias Sociales continúa siendo eminentemente teórica o conceptual, echándose en falta un aprendizaje de carácter más práctico, que vincule lo enseñado a la realidad de las aulas.

Por último, en el primer bloque del cuestionario se interrogó por los cambios que debían introducirse en las prácticas docentes universitarias. Los ítems mejor valorados han sido aquellos vinculados con los enfoques prácticos. Asimismo, una de las propuestas con mayor valoración ha sido la de reforzar la conexión con los centros educativos. En este caso, el 93,3\% de la muestra lo consideró como adecuado o muy adecuado. Otro ítem ampliamente puntuado hace referencia directa a la inclusión de metodologías más activas y prácticas en las aulas universitarias, estando un 95,2\% de acuerdo o muy de acuerdo con introducir este cambio.

Por lo que respecta al segundo objetivo de la investigación, conocer las capacidades y competencias que considera haber adquirido el alumnado egresado participante para la enseñanza de las Ciencias Sociales, destacamos algunos de los resultados más significativos, extraídos del tercer bloque del cuestionario. Respecto a la comparativa entre Grados, cabe señalar que no se han apreciado tendencias significativas en el análisis de datos que evidencien que los participantes de un Grado se sienten más capacitados que los del otro, por tanto, podemos hablar de resultados similares en ambas titulaciones.

En cuanto a la preparación para impartir clases de Ciencias Sociales, un 56,4\% afirma sentirse preparado o muy preparado; un 32,3\% valora su nivel como medio y el $11,3 \%$ restante ha valorado su preparación como baja o muy baja. Estos resultados, pese a ser, en líneas generales, favorables, ya que algo más de la mitad de los participantes consideran buena su preparación, manifiestan que es preciso mejorar la calidad de la formación inicial, así como su impacto en el desarrollo de competencias profesionales en los futuros y futuras docentes que les doten de seguridad para su práctica docente.

El ítem destinado a valorar el nivel de alcance de competencias profesionales requeridas para enseñar Ciencias Sociales arroja que un 51,6\% califica sus competencias como intermedias; un 37,1\% las califican como altas o muy altas; el 8,1\% como bajas; y un 3,2\% como muy bajas. Estos datos, donde solo algo más de un tercio del total considera poseer un nivel de competencias alto o muy alto, demuestran que la formación inicial recibida en esta área no provee, al menos en la opinión de los 
estudiantes egresados, de la suficiente preparación para enfrentar positivamente la enseñanza de las Ciencias Sociales, lo que incide nuevamente en la necesidad de introducir mejoras que transformen esta tendencia.

Es significativo que, pese a este resultado que denota una baja capacitación para enseñar Ciencias Sociales, en el ítem correspondiente a valorar las competencias adquiridas para desarrollar el pensamiento crítico en el alumnado, el futuro profesorado afirma sentirse más seguro y capacitado para ello. Así, el 61,5\% considera disponer de capacidad alta o muy alta; un 25,6\% se manifiesta en niveles intermedios y, por último, el $12,9 \%$ cree que sus capacidades son bajas o muy bajas. Esta diferencia de resultados en relación con el ítem anterior quizás pueda explicarse por el tratamiento trasversal y continuado que se hace del pensamiento crítico en casi todas las materias que cursa el alumnado durante su formación inicial. Una mayor preparación en este sentido iría aparejada a un mayor nivel de capacitación que se observa en estos porcentajes.

Destacamos un último ítem sobre el nivel de seguridad con que cuentan los participantes para enseñar Ciencias Sociales. El análisis de los datos revela que un 54,8\% se siente seguro o muy seguro, que el $29,1 \%$ no se siente ni seguro ni inseguro, y que el 16,1\% muestra niveles bajos o muy bajos de seguridad. Comprobamos que los niveles de seguridad a la hora de enseñar Ciencias Sociales están bastante equilibrados, pues mientras algo más de la mitad de la muestra se siente segura para ello, el resto de los participantes afirman sentirse medianamente seguros o poco seguros; resultados que inciden en la importancia de dotar de mayor preparación y competencias al futuro profesorado de Educación Infantil y Primaria.

\subsection{Resultados del análisis cualitativo}

Para completar los resultados cuantitativos y triangular la información, pasamos a continuación a analizar las entrevistas. Es preciso señalar que por motivos de espacio no hemos incluido la totalidad de resultados alcanzados en ellas, si bien, hemos seleccionado únicamente aquellos datos que atienden a los objetivos de este estudio y que complementan los datos cuantitativos expresados más arriba. Tras la transcripción de las entrevistas y el proceso de análisis de contenido expuesto anteriormente, basado en la codificación y categorización de la información, se identificaron varias categorías y subcategorías recogidas en la Tabla 1.

TABLA 1. Categorías finales

\begin{tabular}{ll}
\hline \multicolumn{1}{c}{ Categorías } & \multicolumn{1}{c}{ Subcategorías } \\
\hline 1.Valoración de la enseñanza & 1.1. Valoración de la formación \\
recibida durante el Grado & 1.2. Valoración del ejercicio docente \\
& 1.3. Utilidad de los saberes recibidos \\
& \multicolumn{1}{c}{ 1.4. Cambios que introducir en la enseñanza } \\
& universitaria recibida \\
\hline 2. Desempeño docente & 2.1. Adquisición de competencias para enseñar \\
& 2.3. Apoyo de compañeros/as \\
& 2.4. Emociones experimentadas \\
\hline 3. Metas que se pretende alcanzar & 3.1. Metas de enseñanza y capacidad para \\
en el ejercicio de enseñanza & alcanzarlas \\
\hline
\end{tabular}

Fuente: elaboración propia

Por lo que respecta a la primera categoría, sobre la valoración de la formación recibida durante el Grado, se aprecian similitudes en las entrevistas, pues ambas entrevistadas aluden a una enseñanza basada en lo teórico y conceptual, resultados que coinciden con los aportados por las encuestas, donde el $75,8 \%$ de la muestra considera la enseñanza recibida en los mismos términos. Sin embargo, sus 
testimonios no coinciden al valorar la utilidad de lo aprendido, pues mientras la docente en ejercicio (E1) considera que no le ha servido de mucho para enfrentarse a la enseñanza de las Ciencias Sociales, la docente que no ha ejercido aún (E2) sí cree que podrá servirle, aunque manifiesta ciertas dudas. Este cuestionamiento de la formación se asimila a lo observado en los resultados de los cuestionarios, donde un 38,7\% se mostraba igualmente escéptico frente a la utilidad de dicha formación. En nuestra opinión, creemos que el hecho de no haber ejercido aún la profesión, podría influir en la valoración que se hace de la formación inicial. En este sentido, la visión negativa que muestra la primera entrevistada (docente en ejercicio), quizás se explique porque ha tenido ocasión de enseñar contenidos sociales y ha comprobado, al menos en su caso, que su formación no ha sido de gran ayuda.

En cuanto a la valoración de la labor docente del profesorado universitario, las dos entrevistadas coinciden en que los/as docentes hacían bien su labor, aunque califican como tediosos algunos elementos como las estrategias metodológicas empleadas para la enseñanza o los contenidos y conceptos abordados. Esta consideración del ejercicio docente se asemeja también a los resultados de las encuestas, en las que casi el $60 \%$ de la muestra calificó como adecuada o muy adecuada dicha labor.

En las entrevistas, al igual que en los cuestionarios, se incluyeron preguntas sobre los cambios que debían introducirse en la formación inicial. Al respecto, las dos entrevistadas concuerdan en que la enseñanza debe ser más práctica, como ocurre en los resultados de las encuestas. Manifestaciones como: "la enseñanza la enfocaría centrándome más en los contenidos que se van a trabajar durante toda la etapa de primaria [...] daría más prioridad a los contenidos prácticos [...]" (E1); o "creo que las Ciencias Sociales tienen que ser más prácticas, porque la teoría no la recuerdas [...]" (E2), muestran que esta transformación se considera indispensable.

La segunda categoría de resultados responde al segundo objetivo específico de esta investigación, relativo a las capacidades y competencias adquiridas para enseñar Ciencias Sociales. En este caso, los resultados de las entrevistas concuerdan con los porcentajes más altos de las encuestas, que se movían entre quienes se sienten preparados para ello $(56,4 \%)$ y quienes no están seguros del todo, posicionándose en valores intermedios (32,3\%). Al primer porcentaje podría asimilarse la entrevistada 2, que afirma: "yo creo que sí me siento preparada para dar clase de Ciencias Sociales" (E2); mientras que al segundo grupo podría asimilarse la entrevistada 1 quien, desde posiciones iniciales dudosas frente a su capacidad para enseñar Ciencias Sociales, busca recursos y logra llevar a cabo su acción docente de modo favorable: "Es verdad que cuando me tocaba dar Ciencias Sociales pensaba «no estás preparada». Pero, cuando he tenido un libro delante y he visto los contenidos, me he puesto a buscar recursos [...] y sí que me he sentido preparada" (E1).

Como última categoría de resultados no incluida en los cuestionarios, pero sí en las entrevistas, tenemos las metas que las docentes pretenden alcanzar con la enseñanza de las Ciencias Sociales. En este sentido, se puede afirmar que el ideal de enseñanza de ambas docentes está relacionado con la búsqueda del protagonismo del alumnado. Aspiran a una enseñanza que sea activa, motivadora y de su interés. Una enseñanza que no se encuentre monopolizada por la utilización de un único recurso. Para ejemplificar dicha opinión se pueden recuperar extractos de las entrevistas, donde la docente novel, por ejemplo, define su ideal de enseñanza del siguiente modo: “[...] mi ideal de enseñanza es el trabajo mediante el juego y mediante actividades lúdicas y divertidas" (E1). En el caso de la docente que no se encuentra ejerciendo, manifestaba lo siguiente: "me gustaría que la educación fuese a partir de los intereses del alumnado [...] quiero que el alumno se equivoque, investigue, se interese [...] quiero que el alumnado disfrute aprendiendo" (E2). Cabe señalar que ese ideal de enseñanza al que hacen referencia es el mismo tipo de enseñanza que demandan en su formación y que se comprueba además en los resultados de los cuestionarios cuando se interroga acerca de qué cambios introducirían los participantes en la docencia universitaria, observándose entonces una mayor demanda de enseñanzas activas y prácticas. 


\section{DiSCUSIÓN Y CONCLUSIONES}

Los resultados obtenidos en esta investigación nos permiten establecer algunos planteamientos para la discusión y algunas conclusiones en relación con cómo valoran los maestros y maestras recién licenciados la formación inicial que han recibido en el ámbito de la Didáctica de las Ciencias Sociales. Somos conscientes de las limitaciones de este estudio, pues sus resultados no son generalizables por el tamaño de la muestra. Asimismo, investigar las percepciones de los futuros docentes en formación, pese a ser valioso y revelador en muchos sentidos, también puede conllevar ciertas limitaciones, como la obtención de respuestas excesivamente racionales y deliberativas, o el análisis de estas concepciones solo desde el punto de vista de lo que se expresa, sin tomar en cuenta otras variables que también influyen (Estellés, Romero y Amo, 2021; Estellés, Amo y Romero, 2021). En nuestro caso, algunas de estas variables podrían ser las concepciones del profesorado en formación inicial sobre las Ciencias Sociales y su enseñanza, o sus propias experiencias con el aprendizaje de esta materia en etapas preuniversitarias.

Si bien, teniendo en cuenta lo dicho, creemos que los resultados alcanzados son significativos por cuanto nos ayudan a reconocer debilidades en la formación inicial del profesorado y a reflexionar sobre posibles mejoras que urge introducir. Asimismo, investigar las prácticas docentes universitarias es una línea de investigación que no ha sido suficientemente explorada (González, 2013), por lo que futuros trabajos similares, extensibles a más muestra y a otros contextos universitarios, permitirán contribuir a un campo de conocimiento esencial para la mejora de la calidad docente y, en consecuencia, de la educación.

Retomando los objetivos de la investigación, podemos señalar que los participantes, en general, poseen una valoración positiva de la enseñanza recibida en Didáctica de las Ciencias Sociales y están contentos con la labor de sus docentes, datos que concuerdan con los de estudios recientes como el de Guerrero, Chaparro y Felices (2020), quienes constatan que el alumnado universitario encuestado en su investigación muestra igualmente una visión favorable de sus profesores y profesoras.

Destacamos además que un porcentaje considerable, un $62,9 \%$, señala que esta formación les ha permitido desarrollar una actitud más crítica. A pesar de ello, la enseñanza se considera esencialmente teórica, por lo que hay una clara reivindicación de estrategias más prácticas, que incorporen el acercamiento a la realidad de las aulas. Estos resultados coinciden con las investigaciones desarrolladas por Van Hover y Hicks (2018), quienes destacan que la enseñanza en el área de Ciencias Sociales ofrecida en la universidad es una enseñanza homogénea y general. Igualmente, concuerdan con los estudios realizados por Guerrero et al. (2020), Lara y Felices (2021) o Arana, Gutiérrez y Aguirre (2021), que constatan en sus trabajos la misma demanda, por parte del alumnado universitario, de nuevas formas de enseñar a enseñar Ciencias Sociales. Estos autores, al igual que apuntara Armento (2000), coindicen en subrayar el distanciamiento existente entre la formación recibida y la realidad de las aulas, un distanciamiento que se hace presente en la entrevista a la docente novel (E1), quien percibe que los aprendizajes adquiridos nada tienen que ver con la práctica educativa de los centros.

En lo que se refiere a los cambios a introducir en los planes formativos, tanto en los cuestionarios como en las entrevistas, se solicita un tipo de enseñanza que sea útil, práctica y que mantenga mayor relación con los contextos educativos de Educación Infantil o Primaria, planteamientos que coindicen con lo expuesto por Pagés (2009), que considera esta enseñanza práctica como un elemento esencial en la formación del profesorado, pues es mediante la implementación de estas metodológicas como se aprende, enfrentando los problemas que pueden surgir en y desde la práctica. Las demandas formativas siguen además la misma línea de Ross (2019), que alude a una formación que permita al profesorado desarrollar una actitud crítica, en la que se les dote de competencias para saber enseñar y no tener que aplicar mecánicamente actividades ya predefinidas. 
Tomando en cuenta el segundo objetivo de nuestra investigación, cuando se interroga por las competencias profesionales y capacidades adquiridas para enseñar Ciencias Sociales, la valoración inicial es buena, sin embargo, es matizable en algunos de los ítems donde los participantes muestran algo más de inseguridad, por ejemplo, a la hora de trabajar interdisciplinarmente las Ciencias Sociales o de conocer el contenido social procedente de las disciplinas a abordar en las aulas. Si bien, pese a las debilidades detectadas en los planes formativos, señaladas por los participantes, lo cierto es que manifiestan sentirse capacitados para enseñar el conocimiento social o para saber dónde pueden encontrar los recursos para hacerlo. Destaca la capacidad que manifiestan poseer para desarrollar el pensamiento crítico del alumnado o para actuar como agentes de trasformación social a través de la enseñanza de las Ciencias Sociales. En este sentido, el 61,3\% de la muestra valora sus capacidades para ello como altas o muy altas. Cabe señalar igualmente que, pese a estos resultados positivos, cuando se les pregunta directamente sobre la valoración que hacen de sus competencias y capacidades para enseñar Ciencias Sociales, los datos varían, pues un 51,6\% considera poseer un nivel medio de competencia, produciéndose aquí un leve cambio de tendencia.

Sin duda, en el proceso de adquisición de competencias profesionales y de formación docente, tienen un peso trascendental tanto los programas formativos que se llevan a cabo en las aulas universitarias, como los docentes encargados de ellos. Estos pueden repercutir significativamente en el alumnado, dotándoles no solo de seguridad y estrategias para trasladar estos enfoques a sus futuras aulas, sino también, para procurar cambios en sus concepciones epistemológicas acerca del conocimiento social, cómo enseñarlo y sus finalidades. En este sentido, la entrevistada 2, la docente egresada que aún no ha ejercido, señalaba en su entrevista que consideraba sentirse capacitada para enseñar Ciencias Sociales porque en uno de los dos años que cursó formación en esta didáctica específica, tuvo una docente que le enseñó a trabajar el conocimiento social en conexión con las problemáticas del entorno, lo que le había procurado un marco conceptual y de actuación adecuado para afrontar la enseñanza en el aula desde este prisma.

Llama la atención que, en las dos entrevistas, las docentes hacen referencia al uso del libro de texto como recurso que les dota de seguridad como sucede en la investigación de Montilla, MaraverLópez, Romero y Martín (2018) quienes, tras indagar en las expectativas de enseñanza del profesorado novel, observaron que un alto porcentaje de los participantes se decantaba por el uso del libro de texto en el aula, ya fuese como instrumento principal o como material de apoyo. La preeminencia del manual no solo se aprecia en el profesorado novel, sino que es una práctica generalizada entre el profesorado que imparte Ciencias Sociales. Así lo demuestran Bel, Colomer y Valls (2016), quienes constataron en uno de sus estudios que era un recurso indispensable en el aula, ya fuese para dar la lección o para realizar actividades.

Tomando en cuenta esta circunstancia, desde la formación inicial debe incidirse en la parcialidad de los manuales y en la ausencia de contenidos que quedan silenciados en los mismos, invisibilizando así parte de la historia, de la geografía y de otras realidades sociales y sujetos. Su empleo exclusivo constriñe, por tanto, la construcción emancipadora y crítica del pensamiento social. Por ello, los contenidos de los libros de texto deben ser analizados e interpretados desde una visión crítica, así como complementados y contrastados con otras fuentes y recursos que favorezcan la construcción de un conocimiento social holístico e inclusivo.

Antes de finalizar, como prospectiva de este estudio, sería interesante aumentar la muestra, tanto para el análisis cuantitativo como cualitativo y abrir la participación a estudiantes del Máster en Profesorado de Educación Secundaria Obligatoria, contexto formativo que precisa igualmente de mejoras (Escudero, Campillo y Sáez, 2019). Debería tenerse en cuenta también la variable del sexo o de los estudios (Grados / Máster), para comprobar si se observan diferencias en cuanto a la valoración de la formación recibida en uno u otro caso. Igualmente importante será investigar las prácticas formativas universitarias desde la visión del propio profesorado que imparte estas materias, e incluir estrategias metodológicas en la línea de la lesson study, que permitan mejorar las prácticas docentes (Calvo, Haya y Rodríguez-Hoyos, 2021; Del Río, 2021). 
Finalmente, a modo de conclusión, podemos destacar algunas implicaciones educativas de este trabajo. Los resultados muestran, en la línea de recientes investigaciones como las de Gomes (2020), que la formación inicial del profesorado en Didáctica de las Ciencias Sociales requiere de transformaciones que incluyan una enseñanza más práctica y conectada con la vida real de las aulas, donde lo trabajado y aprendido en los contextos universitarios pueda ser extrapolado a su ejercicio docente y donde exista una mayor conexión entre lo que el profesorado de los Grados cree hacer y aquello que realmente hace. Asimismo, se requieren planes formativos que refuercen las competencias profesionales de los futuros docentes en relación a qué enseñar y cómo enseñarlo, atendiendo a las finalidades que se otorgan hoy a la enseñanza de las Ciencias Sociales, poniendo énfasis en la formación de profesionales críticos, autónomos, reflexivos y comprometidos, capaces de tomar decisiones y de crear situaciones de aprendizaje que favorezcan el conocimiento social significativo y duradero en su alumnado (Santisteban y Pagès, 2019: 135).

Nuestra investigación y sus resultados llevan a plantear nuevamente necesidades ya detectadas en estudios anteriores que evidencian la importancia de avanzar hacia enfoques críticos en la formación inicial del profesorado, que prioricen los contenidos prácticos y profesionales, y no tanto los conceptuales, y que conecten en mayor medida con la realidad educativa. Solo así podremos dotar al futuro profesorado de las herramientas requeridas para educar en y para la democracia, ayudándoles a reconocer la trascendencia actual de enseñar Ciencias Sociales para construir conocimiento emancipador, que conforme identidades críticas, libres e inclusivas.

\section{Referencias}

Aguilar, S. y Barroso, J. M. (2015). La triangulación de datos como estrategia en investigación educativa. Píxel-Bit. Revista de Medios y Educación, 47, 73-88. DOI: 10.12795/pixelbit.2015.i47.05

Arana, D. M., Gutiérrez, M., C. y Aguirre, C. (2021). De la formación inicial a la enseñanza del conocimiento social del profesorado novel de primaria: una relación por construir. REIDICS, Revista de Investigación en Didáctica de las Ciencias Sociales, 8, 126-140. DOI: 10.17398/2531-0968.08.126

Arana, D. M., Gutiérrez, M. C. y Pagès, J. (2018). Impacto de la formación inicial en las prácticas de profesores noveles que enseñan el conocimiento social en Básica Primaria. En. E. López, C. R. García y M. Sánchez (Eds.), Buscando formas de enseñar: Investigar para innovar en Didáctica de las Ciencias Sociales (pp. 337-346). Valladolid: AUPDCS, Ediciones Universidad de Valladolid.

Aranda, A. M. y López, E. (2017). Logros, dificultades y retos de la docencia e investigación en Didáctica de las Ciencias Sociales. REIDICS, 1, 5-23. DOI: 10.17398/2531-0968.01.5

Armento, B. J. (2000). El desarrollo profesional de los profesores de Ciencias Sociales. En J. Pagès, J. Estepa y G. Travé (Eds.) Modelos, contenidos y experiencias en la formación del profesorado de Ciencias Sociales (pp. 19-39). Huelva: Universidad de Huelva.

Bel, J. C., Colomer, J. C. y Valls, R. (2016). Uso y presencia de los manuales escolares de Ciencias Sociales en Educación Primaria: El caso de la provincia de Valencia. En VV.AA., IV Congreso Internacional de Investigación e Innovación en Educación Infantil y Primaria (pp. 498-503). Murcia: Universidad de Murcia.

Blasco, J. E. y Mengual, S. (2010). Análisis de datos cualitativos asistido por ordenador. En Ciencias de la Educación. En R. Roig Vila y M. Fiorucci (Eds.), Claves para la investigación en innovación y calidad educativas. La integración de las Tecnologías de la Información y la Comunicación y la Interculturalidad en las aulas (pp. 71-84). Alcoy: Editorial Marfil. 
Bonilla-García, M. A., y López-Suárez, A. D. (2016). Ejemplificación del proceso metodológico de la teoría fundamentada. Cinta moebio, 57, 305-315. DOI: 10.4067/S0717554X2016000300006

Calvo, A., Haya, I. y Rodríguez-Hoyos, C. (2021). Aprendiendo el oficio docente. El modelo Lesson Study para mejorar la docencia en la universidad. Educatio Siglo XXI, 39(1), 211-234. DOI: 10.6018/educatio.469241

Chaparro Sainz, A. (2021). Análisis de las prácticas docentes universitarias: ¿qué aprenden nuestros estudiantes en clase? En XXXI Simposio Internacional de Didáctica de las Ciencias Sociales (pp. 1-9). Valencia: Universitat de València, AUPDCS.

Creswell, J. y Plano, V. (2007). Designing and conducting Mixed Methods Research. California: Sage Publications, Inc.

Cuesta, R. (2003). Campo profesional, formación del profesorado y apuntes de didáctica crítica para tiempos de desolación. Didáctica de las Ciencias Experimentales y Sociales, 17, 3-23.

Del Río, J. L. (2021). La Lesson Study como estrategia formativa para aprender a enseñar en la universidad. Algunas reflexiones derivadas de un Proyecto de Innovación Educativa desarrollado en la Universidad Nacional de Educación (UNAE). Revista Complutense de Educación, 32(1), 171-180. DOI: 10.5209/rced.68205

Escudero, J. M., Campillo, M. y Sáez, J. (2019). El Máster de Formación Inicial del Profesorado de Educación Secundaria: Revisión, balances y propuestas de mejora. Profesorado. Revista de curriculum y formación del profesorado, 23(3), 165-188. DOI: 10.30827/profesorado.v23i3.9409

Esteban, F. (2019). La universidad light. Un análisis de nuestra formación universitaria. Barcelona: Paidós.

Estellés, M. (2015). Lo que no vemos sobre la educación ciudadana en la formación del profesorado: supuestos y sobreentendidos. En A. M. Hernández Carretero, C. R. García Ruíz y J. L. De la Montaña Conchiña (Eds.), Una enseñanza de las ciencias sociales para el futuro: Recursos para trabajar la invisibilidad de personas, lugares y temáticas (pp. 483-489). Cáceres: Universidad de Extremadura, AUPDCS.

Estellés, M., Amo, F. J., \& Romero, J. (2021). The Consensus on Citizenship Education Purposes in Teacher Education. Social Sciences, 10(5), 164. DOI: 10.3390/socsci10050164stlle

Estellés, M., Romero, J. y Amo, F. J. (2021). The Paradoxes of Citizenship Education: Frames and Factors Influencing Dispositions toward Discussing Political Issues in the Classroom. Journal of Social Studies Education Research, 12(2), 78-99. DOI: https://jsser.org/index.php/jsser/article/view/2917

Estepa, J. (2017). Otra didáctica de la historia para otra escuela. Lección inaugural del curso 20172018. Huelva: Universidad de Huelva.

George, D. y Mallery, P. (2003). SPSS for Windows step by step: A simple guide and reference. 11.0 update (4th ed). Boston: Allyn \& Bacon.

Gomes, A. (2020). História e da Didática da História: práticas e conceções na formação inicial de professores do ensino básico (10-12 anos). Enseñanza de las Ciencias Sociales, 19, 5-16. DOI: 10.1344/ECCSS2020.19.2

Gómez-Carrasco, C. J., López-Facal, R. y Rodríguez-Medina, J. (2019). La investigación en Didáctica de las Ciencias Sociales en revistas españolas de Ciencias de la Educación. Un análisis bibliométrico (2007-2017), Didáctica de las Ciencias Experimentales y Sociales, 37, 67-88. DOI: 10.7203/DCES.37.14440

Gómez, C. J., Souto, X. M. y Miralles, P. (Eds.) (2021). Enseñanza de las ciencias sociales para una ciudadanía democrática. Estudios en homenaje al profesor Ramón López Facal. Barcelona: Octaedro.

González, G. A. (2012). Formación inicial del profesorado y educación para la ciudadanía en Colombia: representaciones sociales y prácticas de enseñanza. Barcelona: UAB. 
González, G. A. (2013). La formación inicial del profesorado y la educación para la ciudadanía: representaciones sociales, diseño de clases y prácticas de enseñanza. Enseñanza de las Ciencias Sociales, 12, 37-45.

González, M. y Fuentes, E. J. (2011). El Practicum en el aprendizaje de la profesión docente. Revista de Educación, 354, 47-70.

Guerrero, R., Chaparro, Á. y Felices, M. M. (2020). La práctica docente en Didáctica de las Ciencias Sociales a revisión: una investigación en el Grado en Educación Primaria. En E. LópezMeneses, D. Cobos-Sanchiz, L. Molina-García, A. Jaén-Martínez y A. H. Martín-Padilla (Eds.), Claves para la innovación pedagógica ante los nuevos retos (pp.1574-1582). Barcelona: Octaedro.

Hernández, F. y Maquilón, J. J. (2010). Introducción a los diseños de investigación educativa. En S. Nieto (Ed). Principios, métodos y técnicas esenciales para la investigación educativa (pp. 109126). Madrid: Dykinson.

Iranzo, P. (2018). ¿Qué opinan los Maestros sobre las Competencias de Liderazgo Escolar y sobre su Formación Inicial?, REICE. Revista Iberoamericana sobre Calidad, Eficacia y Cambio en Educación, 16(3), 29-48. DOI: 10.15366/reice2018.16.3.002

Lara, S. y Felices, M. M. (2021). Investigar la formación inicial del profesorado. Resultados para la reflexión. En M. D. C. Sánchez, J. M. Campillo y V. Vivas (Eds.), La formación del profesorado en didáctica de las ciencias sociales en el ámbito Iberoamericano (pp. 439-452). Murcia: Editum.

Marcelo, C. (1991). Aprender a Enseñar: un estudio sobre el proceso de socialización de profesores principiantes. Madrid: C.I.D.E.

Martínez, R., Castellano, M. A. y Chacón, J. C. (2014). Métodos de investigación en psicología. Madrid: EOS Universitaria.

McMillan, J. H. y Schumacher, S. (2005) Investigación educativa. Madrid: Pearson Educación, $5^{\text {a }}$ Ed.

Montero, L., Martínez, E. y Colén, M. (2017). Los estudios de Grado en la formación inicial de Maestros en Educación Primaria. Miradas de formadores y futuros maestros. Profesorado. Revista de Currículum y Formación de Profesorado, 21(1), 1-16.

Montilla, M. V. C, Maraver-López, P., Romero, C. y Martín, A. (2018). Análisis de las expectativas del profesorado novel sobre su futura labor docente. Aula abierta, 47(2), 185-192. DOI: 10.17811/rifie.47.2.2018.185-192

Moreno, O. (2015). "Lo que hoy no te enseñaremos". El profesorado ante la enseñanza de cuestiones relevantes en las aulas. En A. M. Hernández, C. R. García y J. L. De la Montaña (Eds.), Una enseñanza de las ciencias sociales para el futuro: Recursos para trabajar la invisibilidad de personas, lugares y temáticas (pp. 917-924). Cáceres: Universidad de Extremadura, AUPDCS.

Moril, R. (2011). Buenas prácticas en la didáctica de las ciencias sociales. Revista EDETANIA, 40, 173-187.

Pagès, J. (2009). Enseñar y aprender ciencias sociales en el siglo XXI: reflexiones casi al final de una década. En Investigación en Educación, Pedagogía y Formación Docente, II Congreso Internacional (pp. 140-154). Medellín: Universidad Pedagógica Nacional, Universidad de Antioquia, Corporación interuniversitaria de servicios.

Pagès, J. (2019). Ciudadanía global y enseñanza de las Ciencias Sociales: retos y posibilidades para el futuro. REIDICS: Revista de Investigación en Didáctica de las Ciencias Sociales, 5, 5-22. DOI: $10.17398 / 2531-0968.05 .5$

Pagès, J. y Santisteban, A. (2014). Una mirada desde el pasado al futuro en la didáctica de las Ciencias Sociales. En J. Pagés, A. Santisteban (Eds.), Una mirada al pasado y un proyecto de futuro. Investigación e innovación en Didáctica de las Ciencias Sociales (pp. 17-39). Barcelona: UAB, AUPDCS. 
Parra, D. y Fuertes, C. (Coords.) (2019). Reinterpretar la tradición, transformar las prácticas: Ciencias Sociales para una educación crítica. Valencia: Tirant Lo Blanch.

Pérez, Á. I. (2019). Ser docente en tiempos de incertidumbre y perplejidad. Márgenes Revista De Educación De La Universidad De Málaga, 0(0), 3-17. DOI: 10.24310/mgnmar.v0i0.6497

Piñuel, J. L. (2002). Epistemología, metodología y técnicas del análisis de contenido. Estudios de Sociolingüistica, 3(1), 1-42.

Rey, B. (2007). Les compétences professionnelles dans la transmission scolaire du savoir historique. En R. M. Ávila, J. R. López y E. Fernández (Eds.) Las competencias profesionales para la enseñanza-aprendizaje de las ciencias sociales ante el reto europeo y la globalización (pp. 3354). Bilbao: AUPDCS.

Ross, W. (2019). The Challenges of Teaching Social Studies: What Teachers? What Citizenship? What Future? En M. Ballbé, N. González-Monfort y A. Santisteban (Eds.). Quin professorat, quina ciutadania, quin futur? Els reptes de l'ensenyament de les ciències socials, la geografia i la historia (pp. 39-52). Barcelona: GREDICS, UAB.

Sanmartí, N. (2007). La formació dels professors de secundària: quin "model organitzatiu" de formació?. Grup d'Opinió sobre el Sistema Educatiu. La formació inicial del professorat de secundaria (pp. 53-62). Barcelona: ICE-UB.

Santisteban, A. (2015). La formación del profesorado para hacer visible lo invisible. En A. M. Hernández, C. R. García y J. L. De la Montaña (Eds.), Una enseñanza de las Ciencias Sociales para el futuro: recursos para trabajar la invisibilidad de personas, lugares y temáticas (pp. 383-393). Cáceres: Universidad de Extremadura, AUPDCS.

Santisteban, A. (2019). La enseñanza de las Ciencias Sociales a partir de problemas sociales o temas controvertidos: estado de la cuestión y resultados de una investigación. El Futuro del Pasado, 10, 57-79. DOI: 10.14516/fdp.2019.010.001.002

Santisteban, A. y Pagès, J. (2019). Una nueva lectura de los programas de estudios para la formación inicial del profesorado de didáctica de las Ciencias Sociales. Mirando el presente y el futuro. En M. J. Hortas, A. Gomes y N. de Alba (Coords.), Enseñar y aprender didáctica de las Ciencias Sociales: la formación del profesorado desde una perspectiva sociocrítica (pp. 128138). Lisboa: Ediciones Escola Superior de Educação de Lisboa.

Vaillant, D. y Marcelo, C. (2015). El ABC y D de la Formación Docente. Madrid: Narcea.

Van Hover, S. y Hicks, D. (2018). History teacher preparation and professional development. In S. A. Metzger \& L. M. Harris (Eds.). The Wiley International Handbook of History Teaching and Learning (pp. 391-418). Medford, MA: Wiley Blackwell. DOI:10.1002/9781119100812.ch15

\section{CÓMO CITAR ESTE ARTÍCULO}

Darouech, S y Felices de la Fuente, M. M. (2021). La formación inicial en Didáctica de las Ciencias Sociales según el alumnado egresado. Didáctica de las ciencias experimentales y sociales, 41, 5772. DOI: $10.7203 /$ DCES.41.21294 
